\title{
A METAMODELLING IMPLEMENTATION OF A TWO-WAY COUPLED MESOSCALE-MICROSCALE FLOW MODEL FOR URBAN AREA SIMULATIONS
}

\author{
G. Tsegas, Ph. Barmpas, I. Douros and N. Moussiopoulos
}

Laboratory of Heat Transfer and Environmental Engineering (LHTEE), Aristotle University, Thessaloniki, Greece

\begin{abstract}
Systems of coupled prognostic mesoscale and microscale models have been used as a tool to accurately simulate flows around artificial structures and over densely-built urban areas. Typical implementations of such systems are based on a one-way coupling scheme, where the mesoscale model provides initial and boundary conditions for each off-line application of the microscale model. While very successful in predicting steady-state flows within specific local-scale areas, such schemes fail to account for feedbacks on the mesoscale flow induced by the presence of structures in smaller scales. Unfortunately, the large gap of spatial and temporal scales practically prohibits parallel on-line execution of the mesoscale and microscale models for any significant time interval. It is therefore necessary that a simplifying approach is adopted, where the microscale feedback is spatially and temporally upscaled to interact with parts of the mesoscale domain covering the urban area. In the present work a two-way coupled model system is developed, consisting of the prognostic mesoscale model MEMO and the microscale model MIMO. The microscale feedback on the mesoscale domain is simulated using a metamodelling approach, where the effect of local flows on the vertical profiles is estimated for representative urban areas of sizes up to a few hundred meters and used as calibration input for a set of interpolating metamodels. The feedback from the microscale metamodels is then introduced back in the mesoscale grid by means of Newtonian relaxation. As an illustrative application, simulations for the city of Athens, Greece during a multi-day period are presented. Effects of the microscale feedback on the mesoscale flow become evident both as a reduction of lower-level wind speeds in urban cells as well as an overall increase in turbulent kinetic energy production over densely-built areas.
\end{abstract}

Key words: metamodelling, urbanisation, mesoscale, microscale, two-way coupling.

\section{INTRODUCTION}

Efforts to introduce urban effects on mesoscale models have in general followed two different approaches. In the first one, corrections are applied in the mesoscale parameterisations within the lower computational layers, in an attempt to account for the specific characteristics of the urban canopy (Baklanov, 2004). Improved parameterisations include the effects of shadowing and radiation trapping as well as the urban heat island effect (Grimmond and Oke, 1999). The effect of urban canopy on the momentum flow and turbulent kinetic energy (TKE) production is usually parameterised as additional aerodynamic drag and production terms, respectively, introduced in the dynamical equations (Dupont et al., 2004). The so-called "urbanisation" approaches have the advantage of relatively low computational requirements and offer a large degree of flexibility in adjusting the relative strength of the forcing terms, allowing a fine-tuning to the particular characteristics of different urban morphologies. On the other hand, the number of introduced parameters is often quite large and a significant effort needs to be exerted in order to adjust physically sound values, valid for a wide range of urban canopy characteristics such as building height and geometry, density of street canyons and thermal properties of the urban elements.

More recently an alternative approach was proposed, based on Computational Fluid Dynamics (CFD) calculations or wind tunnel measurements of flows over arrays of symmetric obstacles, meant to represent areas of dense urban fabric with varying configurations of street canyon geometries (Coceal and Belcher, 2004; Martilli and Santiago, 2007). In an effort to upscale the calculated (or measured) effect of the obstacle array on the area-averaged drag force and TKE profiles, appropriate parameterisations are again introduced in the larger scale model, taking into account a number of morphological parameters of the array (Bou-Zeid et al., 2007). Since the number of free parameters is usually reduced compared to a full urbanisation scheme, CFD-based methods alleviate the need for calibration runs on the larger scales and/or intricate physical schemes for fixing the parameter values. Yet again, the transferability of such methods depends on both the extent to which various flow configurations are adequately sampled during the calculations (or measurements) and on the sufficient characterization of the city morphology by the chosen parameterizations (Martilli and Santiago, 2007).

In the present work, a novel scheme is developed for introducing effects of the urban canopy calculated on the microscale to a mesoscale calculation, by means of two-way coupling between the mesoscale MEMO model (Moussiopoulos et al., 1993) and the microscale model MIMO (Ehrhard et al., 2000). One-way coupling of mesoscale-to-microscale systems was initially devised as a way to provide microscale calculations with accurate initial and boundary conditions aiming to improve the accuracy of flow computations within and around elements of the urban canopy (Kunz et al., 2000). On the other hand, a two-way coupling scheme would ideally be able to account for the effect of the urban structures, to the extent represented by the microscale calculations, on the calculated mesoscale flow (Martilli, 2007). The main obstacle for the implementation of such a coupled system would be the large gap of spatial and temporal scales between the two models, as well as a prohibitive gap in their execution speeds: the smallest practical mesoscale grid could cover an area of the order of a few tens of kilometres with a grid resolution of $500 \mathrm{~m}$, using a timestep on the order of 2 to $10 \mathrm{~s}$, while typical calculation times for a 24hour simulation is of the order of tens of minutes. For comparison, a building- or neighbourhood-scale CFD case 
could reach a maximum spatial extent of about $1500 \mathrm{~m}$ while the calculation of the steady flow for a single set of boundary conditions would need several minutes, even hours to complete.

In the following sections, the proposed metamodelling scheme, aiming to approximate the time-consuming microscale calculations with a simple interpolating model during the course of the two-way coupled run, is described. The performance of the coupled MEMO-MIMO model is assessed using a springtime case for the greater area of Athens, Greece.

\section{METHODOLOGY}

Metamodelling techniques are increasingly used in simulation applications where full execution of a complex model for many different input conditions would be computationally expensive (Hendrickx and Dhaene, 2005). In effect, a metamodel aims to replace complex modelling of a deterministic system by a cheap numerical interpolating scheme which has been calibrated by sampling the original model's input and output (Piñeros Garcet et al., 2006).

The purpose of the present metamodel is to simulate in a first approximation the area-averaged outflow response of each microscale domain to different average inflow profiles without performing a full microscale calculation at each timestep of the coupled model. Its operation is based on a multi-dimensional interpolator of the form:

$$
\psi(z)=\frac{\sum_{k} R_{k}(z) \cdot \psi_{k}(z)}{\sum_{k} R_{k}(z)}
$$

where $\psi(z)$ denotes vertical profiles of quantities that depends on the relation between outbound and inbound profiles of each physical parameter and $\psi_{k}(z)$ denotes the corresponding calibration values. In the case of inflow-outflow velocities, the interpolated quantities are the velocity ratios $\psi(z)=u_{\text {out }}(z) / u_{\text {in }}(z)$ and $\psi_{k}(z)=u_{\text {out }}^{k}(z) / u_{\text {in }}^{k}(z)$, defined for the same height $z$ for opposing boundaries, as indicated in Figure 1 (middle). In this case, the weighting coefficients are defined as:

$$
R_{k}(z)=\left[u_{\text {in }}(z)-u_{\text {in }}^{k}(z)\right]^{-2}
$$

Similar relations were defined for other flow parameters, such as the deflection angle between the average inflow and outflow directions, and the microscale contribution on the TKE production profile.

Having established an interpolation method, a spatial and temporal sampling of microscale cases is required in order to assign calibrated metamodel instances to all of the microscale areas that will be included in the coupling scheme. As a first step, a small number of specific urban areas of high building density are chosen as representative of the urban geometry of the entire urban area and subsequently used in the actual microscale simulations. In addition, specific hours of the mesoscale simulation period are selected for the microscale cases, as to represent different inflow and atmospheric stability conditions (Figure 1, left). In the next step, microscale calculations are performed for these template cases during which area-averaged inflow and outflow profiles are extracted from the calculated fields for each representative area. The calculated profiles are subsequently used as calibration inputs for the interpolating metamodel (Fig. 1, middle). Multiple instances of the metamodel are calibrated, one for each representative urban area. Finally, an array of metamodel instances, each representing the microscale effects on one mesoscale urban grid cell (Figure 1, right), is set up to interact with the mesoscale calculation by means of Newtonian relaxation (nudging). In this way, the microscale effect is assimilated back into the mesoscale domain via the metamodel in the form of an additional forcing term in the dynamical equations.

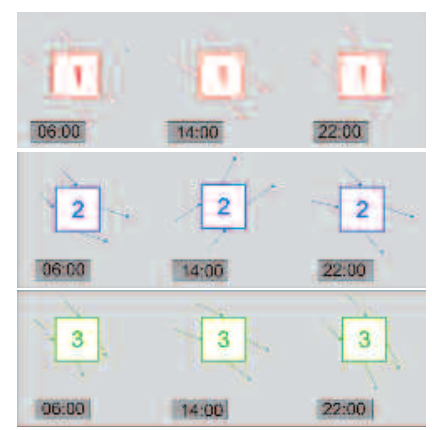

\section{Microscale Domain Mesoscale Domain}
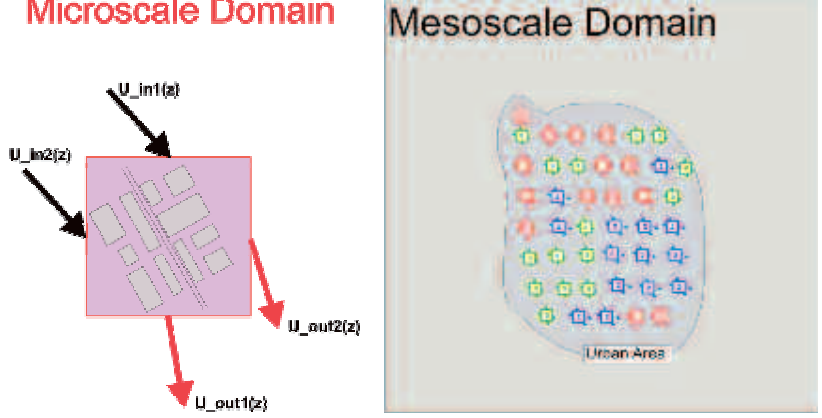

Figure 1. Spatial and temporal sampling of microscale cases inside the urban area.

\section{APPLICATION: THE ATHENS CASE STUDY}

The performance of the coupled MEMO-MIMO model system was assessed using a five-day case between 8 May and 12 May 2002 for the greater Athens area. The aims of the case study were twofold: firstly, to evaluate the performance of the interpolating metamodel for the range of inflow velocity and angle profiles provided by an actual 
mesoscale calculation. Secondly, an effort was made to adjust the value of the relaxation constant $g$ of the Newtonian relaxation so as to optimise the effect of the coupling without introducing numerical instabilities or artifacts in the mesoscale domain. The proposed values for $g$ usually range from $10^{-3}$ to $10^{-1}$ while the optimum value in the Athens case calculations was estimated to be $10^{-2}$. Following the standard mesoscale parameterization of surface roughness over urban areas, the value of the aerodynamic roughness $z_{0}$ would be set to an increased value in the case of urban cells. Nevertheless, the introduction of coupling with microscale tends to externally induce additional drag and turbulence production effects for the same cells. Therefore, a lower value of $z_{0}$ was used in the urban cells of the coupled system, while comparisons were made to standard mesoscale calculations based on the increased urban $z_{0}$.

The application of the coupled system proceeded along the steps outlined in the previous section, for each one of its components, as described below.

\section{Mesoscale model}

An initial mesoscale calculation was performed with MEMO, in order to provide an approximation of the wind flow over the simulated period and to determine the necessary boundary conditions for the microscale simulations during the sampling periods. Two computational grids were used in a nested configuration: a coarse grid with an extent of $100 \times 100 \mathrm{~km}^{2}$ and resolution of $2 \mathrm{~km}$, covering the major part of the Attica peninsula, and a fine grid extending to $36 \times 36 \mathrm{~km}^{2}$ with $500 \mathrm{~m}$ resolution, covering the Athens urban area. Initial and lateral boundary conditions for the outer grid were obtained using meteorological sounding data.

\section{Microscale model}

Two representative areas with linear dimensions of approximately $400 \mathrm{~m}$ were selected for the microscale cases used as a calibration. The first area, located near the shore of Piraeus, represents a typical case of densely-built urban seafront with multi-store buildings, numerous urban canyons and a large open area at the middle. The second selected area, centred around Patision and Fokionos Negri streets in downtown Athens, is an example of very densely built area with poor ventilation along its lateral boundaries and an orthogonal configuration of roads carrying a significant traffic load during most of the day. In order for both areas to be simulated in the microscale, appropriate computational grids were constructed based on 3-dimensional geometrical data of the building and road structure. The increased spatial extent of the grids of both domains led to a rather large total number of cells, of the order of $5 \times 10^{6}$ (Fig. 2, left).

A total of four sampling periods were selected from the case period, to be used for the actual microscale simulations and the subsequent calibration of the metamodel. Table 1 summarises the prevailing conditions for each one of the sampling periods.

Table 1. Microscale sampling time periods defined for the Athens case.

\begin{tabular}{|l|l|l|l|l|l|l|l|l|}
\hline \multirow{2}{*}{ Date and time (LST) } & \multicolumn{2}{l|}{$\begin{array}{l}\text { Average wind } \\
\text { direction }\end{array}$} & \multicolumn{2}{l|}{$\begin{array}{l}\text { Average wind speed } \\
\text { at } 10 \mathrm{~m}\left(\mathrm{~ms}^{-1}\right)\end{array}$} & \multicolumn{2}{l|}{$\begin{array}{l}\text { Average tempera- } \\
\text { ture at } 10 \mathrm{~m}\left({ }^{\circ} \mathrm{C}\right)\end{array}$} & \multicolumn{2}{l|}{ Atmospheric Stability } \\
\cline { 2 - 9 } & Piraeus & Patision & Piraeus & Patision & Piraeus & Patision & Piraeus \\
\hline $08 / 05 / 2002: 11: 00$ & NE & NE & 5.5 & 4.9 & 21.3 & 21.9 & Very unstable & Very unstable \\
\hline $09 / 05 / 2002: 12: 00$ & SE & SE & 3.2 & 3.6 & 19.9 & 22.2 & Very unstable & Very unstable \\
\hline $11 / 05 / 2002: 09: 00$ & WSW & WNW & 4.0 & 3.5 & 20.5 & 21.6 & Unstable & Very unstable \\
\hline $11 / 05 / 2002: 16: 00$ & SW & SW & 3.7 & 3.7 & 22.7 & 25.0 & Unstable & Unstable \\
\hline
\end{tabular}
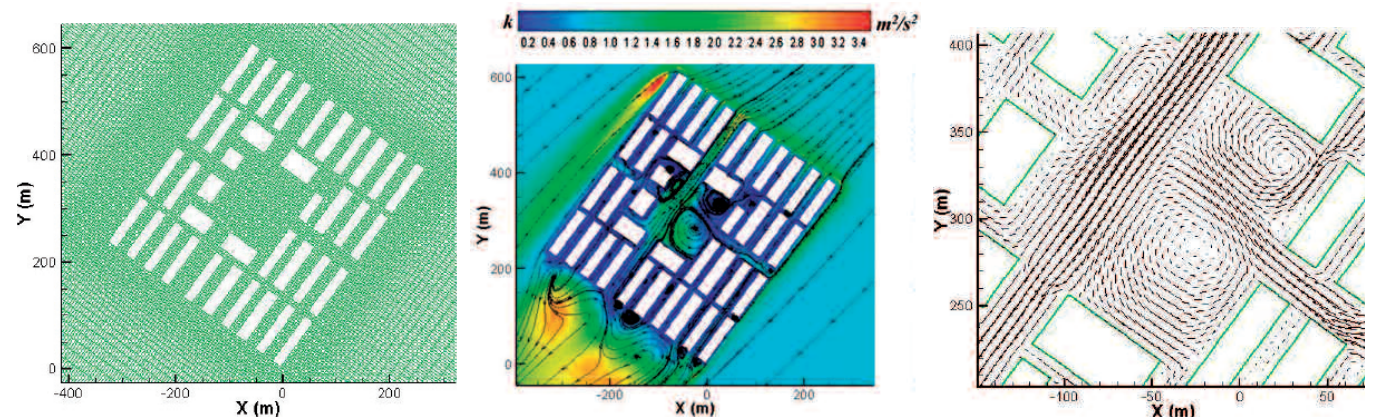

Figure 2. Structured grid (left) and calculated flow fields (middle \& right) over the Piraeus domain for 08/05/2002:11:00 LST. The color map represents calculated TKE values.

Typical calculated flow snapshots, corresponding to a height of about $15 \mathrm{~m}$ over the sample area of Piraeus, are shown in Figure 2. Of particulate interest is the overall decrease of TKE within narrow street canyons and densely built areas, which comes in agreement with previous computational and experimental studies that demonstrated a significant deceleration of the incoming flow and subsequent decrease of the TKE production as it approaches dense 
arrays of high obstacles (Santiago et al., 2007). A slight increase of TKE is observed only within the large open area at the center of the domain, where the flow profiles tend to resume their unperturbed upstream form.

\section{Calibration of the metamodel}

Using as input the area-averaged inflow and outflow profiles of the microscale simulations over the four sampling periods, two separate metamodel instances were calibrated for the Patision and the Piraeus sample areas, respectively. As outlined in the previous section, the two metamodel configurations were transferred to the rest of the urban cells of the mesoscale grid using appropriate horizontal rotations and vertical scaling to account for differences in street orientation and average building height. Based on an elementary classification scheme, urban cells of average-to-high building height located near the sea front were assigned to the Piraeus metamodel configuration, while the rest of the cells were assigned to the metamodel calibrated for Patision St. In order to estimate the angle of rotation and vertical scaling factor for each $500 \times 500 \mathrm{~m}^{2}$ cell, the prevailing street orientation and average building height were determined for each cell, based on aerial photographs in conjunction with a high resolution elevation map. The resulting maps are shown in Figure 3 where red lines indicate the "primary" average street orientation (defined as the one closer to the prevailing direction in the neighbourhood of the cell) while the green lines indicate the "secondary" average orientation (normal to the prevailing direction of adjacent cells).
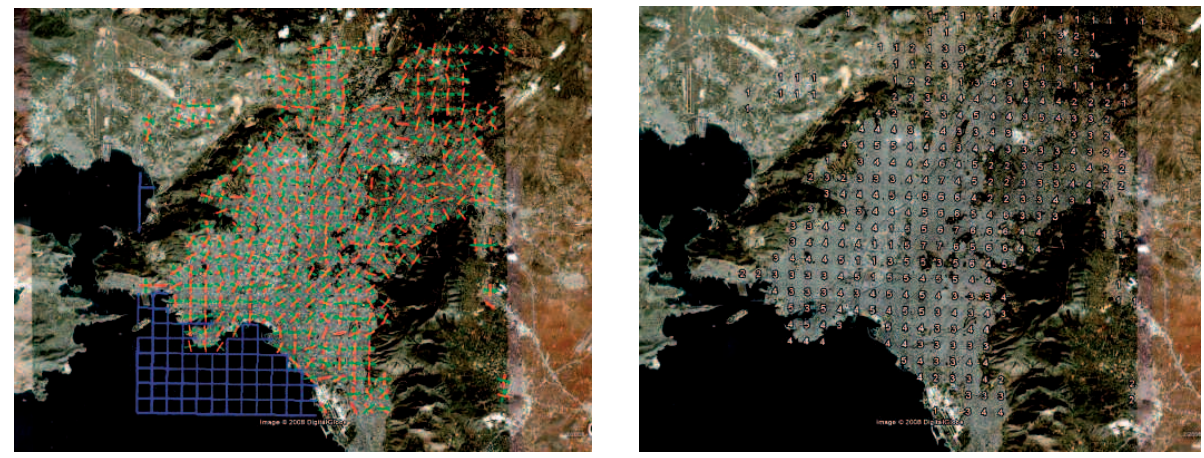

Figure 3. Maps of prevailing street direction and average number of building stories.

\section{Mesoscale calculation with coupling}

In the final mesoscale calculation, the effect of the microscale metamodel was introduced back to the mesoscale domain by means of Newtonian relaxation, which was applied at each mesoscale computational step. Figure 4 shows difference maps between the average wind velocity and TKE fields calculated by the coupled system and the corresponding fields calculated by a standard MEMO simulation. With both model versions, a period of 72 hours starting on 08/05/2002 at 00:00 was used for the time averaging. Looking at the wind velocity map for the first layer $(0-20 \mathrm{~m})$ as show in Figure 4 (left), a significant reduction of about $0.5-1.0 \mathrm{~ms}^{-1}$ becomes apparent over the entire urban area of the domain, where the nudging was applied. The slight increase along the southern coastline is probably caused by the increased velocities on the second computational level (20-43m; Figure 4, middle), which in turn is associated with the appearance of an internal boundary layer over the urban region. Finally, a significant increase of the TKE production is observed in the first three computational levels but becomes more pronounced on the second level (Figure 4, right), in line with the corresponding increase of wind speeds at this height. The TKE increase should be interpreted as a result of both additional wind shear, introduced in the two lower levels by the momentum nudging, as well as the direct influence of the TKE nudging terms in the same levels.
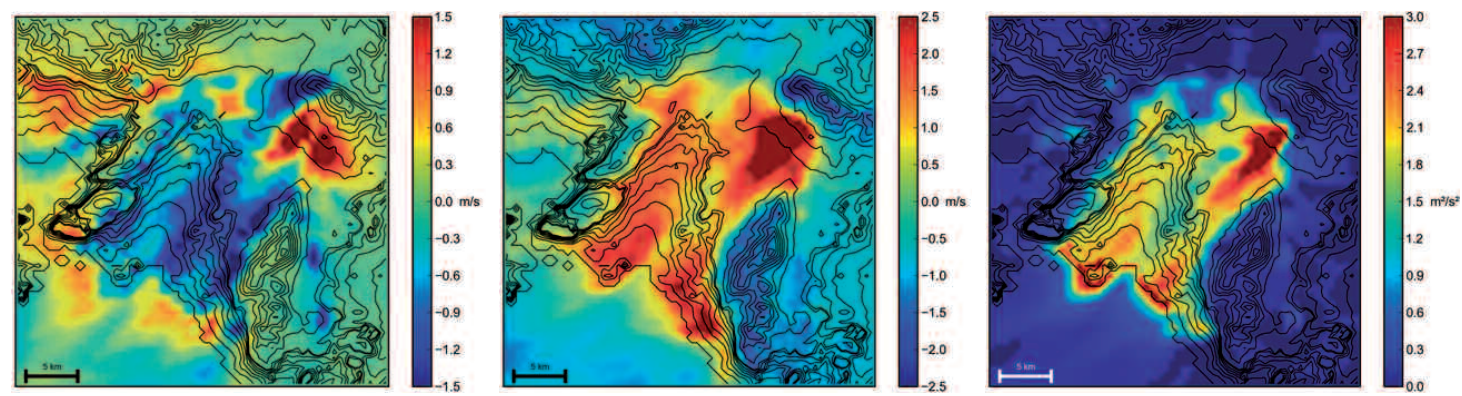

Figure 4. Difference map of the calculated average wind velocities on the first (left) and second (middle) computational layer, and average TKE on the second computational level (right).

Calculated time series of temperature, wind speed and wind direction for a location inside the representative area of Patision St. are shown in Figure 5, together with a wind rose obtained for the 6-day simulation period. For almost the 
entire period, the wind velocity calculated by the coupled system is significantly reduced compared to the stand-alone MEMO run. The angular distribution of wind direction indicates a tendency of the metamodel to align the mesoscale flow with the prevailing street direction of the particular urban cell, in this case NNE-SSW. A small secondary peak along the ESE direction is probably related to streaming effects along the secondary direction (WNW-ESW) for the same cell.

In comparing the values calculated by the coupled system with measurements, the location of the particular meteorological station should be taken into account: since it is installed about $4 \mathrm{~m}$ higher than the roof height of the particular area, the effect of streaming and deceleration within the street canyons would appear diminished. For a more thorough validation of the system, comparison with a set of combined roof- and street-level measurements would be more appropriate, since it would tend to reveal local canalisation effects over heights comparable to the height of the first level of the mesoscale model. Both calculated temperature series show good agreement with measurements, with the exception of the first 24 hours when MEMO was unable to reproduce the overcast conditions that prevailed over the Attica peninsula.

\section{CONCLUSIONS}

A metamodelling methodology was developed for the implementation of an efficient two-way coupling between a mesoscale and a microscale CFD model for calculations over extended densely-built urban areas. Local forcings, obtained from microscale calculations over selected time periods for specified sample urban areas, are used as a calibration input for an
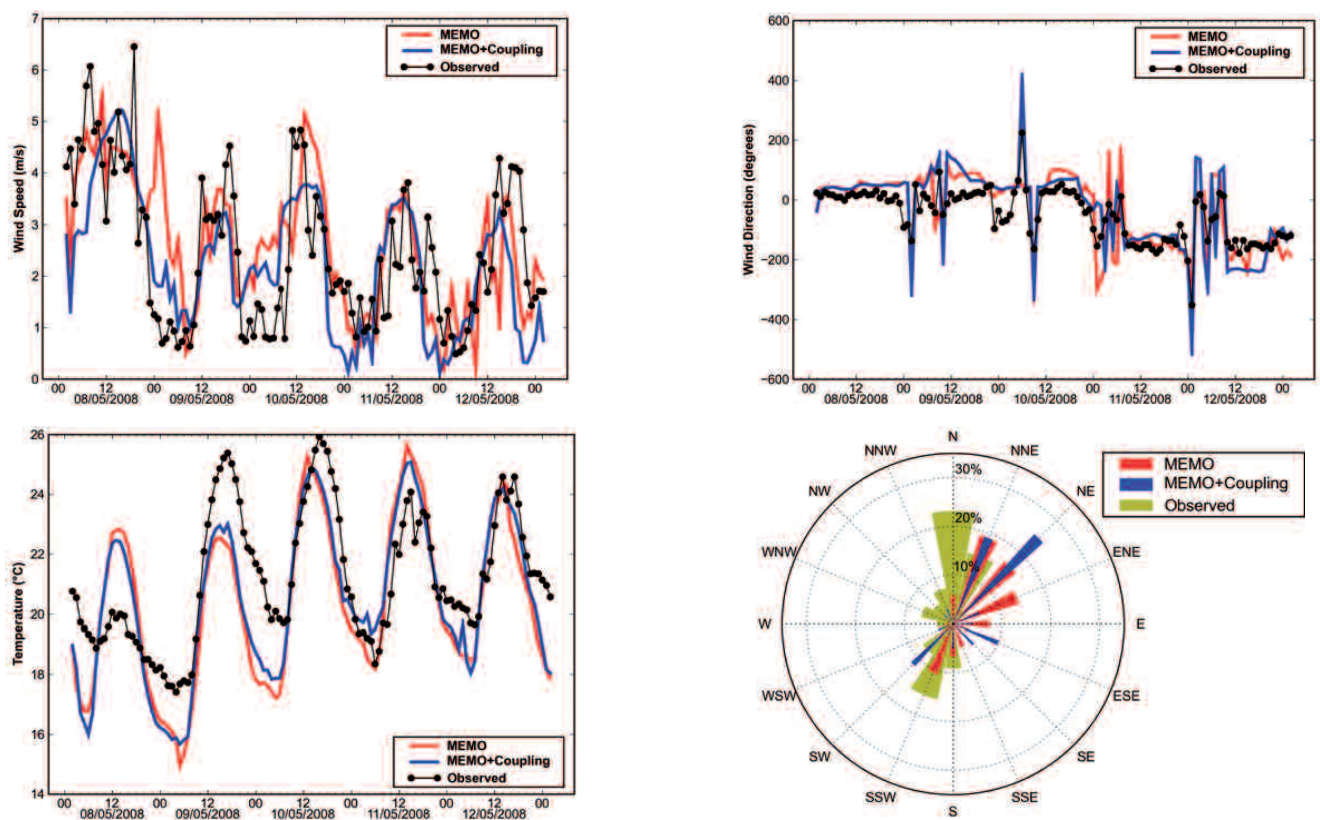

Figure 5. Calculated time series of wind velocity (upper left), wind direction (upper right), temperature (lower left), and windrose (lower right) for the Patision station.

interpolating metamodel. After a suitable discretisation and categorisation of the extended urban area on the mesoscale, metamodel instances are assigned to each of the mesoscale urban cells. During the coupled stage of the run, the metamodel output is assimilated back in the mesoscale field by means of Newtonian relaxation.

Results from the microscale simulations for the sample urban areas reveal an overall reduction in flow velocities within areas containing narrow street canyons as well as a relaxation of the profiles calculated over wide open areas to their unperturbed form.

The model system was applied to a mesoscale case covering the urban area of Athens and the results were compared to the traditional approach of modifying $z_{0}$ over urban areas. It was found that the coupled system on average predicts lower wind velocities over most of the urban cells while the production of turbulent kinetic energy becomes pronounced at the second model layer $(30-40 \mathrm{~m})$. The distribution of wind directions calculated for a downtown location indicates a tendency to align local flows with the prevailing street direction. 


\section{REFERENCES}

Baklanov, A., 2004: European FUMAPEX project: Integrated Systems for Forecasting Urban Meteorology, Air Pollution and Population Exposure, EURASAP Newsletter, 52, 6-36.

Bou-Zeid, E. and M.B. Parlange, 2007: On the Parameterization of Surface Roughness at Regional Scales. $J$. of the Atm. Sci., 64, 216-227.

Coceal, O. and S.E. Belcher, 2004: A canopy model of mean winds through urban areas. Quarterly Journal of the Royal Meteorological Society, 130, 1349-1372.

Dupont, S., T.L. Otte and J.K.S. Ching, 2004: Simulation of meteorological fields within and above urban and rural canopies with a mesoscale model (MM5). Boundary-Layer Meteorolology, 113, 111-158.

Ehrhard, J., I.A. Khatib, C. Winkler, R. Kunz, N. Moussiopoulos and G. Ernst, 2000: The microscale model MIMO: Development and assessment. Journal of Wind Engineering and Industrial Aerodynamics, 85, 163-176.

Grimmond, C.S.B. and T.R. Oke, 1999: Heat storage in urban areas: local-scale observations and evaluation of a simple model. Journal of Applied Meteorology, 38, 922-940.

Hendrickx, W. and T. Dhaene, 2005: Sequential design and rational metamodelling. in Proceedings of the 2005 Winter Simulation Conference (Kuhl, M.E., N.M. Steiger, F.B. Armstrong and J.A. Joines, Eds.), 290-298.

Kunz, R., I. Khatib and N. Moussiopoulos, 2000: Coupling of mesoscale and microscale models - an approach to simulate scale interaction. Environmental Modelling \& Software, 15, 597-602.

Martilli, A. and J.L. Santiago, 2007: CFD simulation of airflow over a regular array of cubes. Part II: analysis of spatial average properties. Boundary-Layer Meteorology, 122, 635-654.

Martilli, A., 2007: Current research and future challenges in urban mesoscale modeling. Int. J. of Clim., 27, 1909-1918.

Moussiopoulos, N., Th. Flassak, D. Berlowitz and P. Sahm, 1993: Simulations of the Wind Field in Athens With the Nonhydrostatic Mesoscale Model MEMO. Environmental Software, 8, 29-42.

Piñeros, Garcet, J.D., A. Ordoñez, J. Roosen and M. Vanclooster, 2006: Metamodelling: Theory, concepts and application to nitrate leaching modelling. Ecological Modelling, 193, 629-644.

Santiago, J.L., A. Martilli and F. Martin, 2007: CFD simulation of airflow over a regular array of cubes. Part I: 3-D simulation of the flow and validation with wind tunnel data. Boundary-Layer Meteorology, 122, 609-634. 